\title{
Level Sets of Multiparameter Stable Processes
}

\author{
Thomas S. Mountford ${ }^{1}$
}

Received February 14, 2005; revised September 27, 2005

We establish the correct Hausdorff measure function for the level sets of additive strictly stable processes derived from strictly stable processes satisfying Taylor's condition (A). This leads naturally to a characterization of local time in terms of the corresponding Hausdorff measure function of the level set.

KEY WORDS: Local times; Hausdorff measure; level sets; additive stable processes.

AMS 2000 Subject Classifications: Primary: 60G60; Secondary: 60G15, $60 \mathrm{G} 17$.

\section{INTRODUCTION AND RESULTS}

In this article, we wish to extend the approach of Ref. 7 to additive (or multi-parameter) $\alpha$-stable processes $(0<\alpha<2) \underline{X}: I R^{N} \rightarrow I R^{d}$ given by

$$
\underline{X}\left(t_{1}, \ldots, t_{N}\right)=\sum_{j=1}^{N} X_{j}\left(t_{j}\right),
$$

where $\left(X_{j}(t):-\infty<t<\infty\right)_{i=1}^{N}$ are independant two sided-strictly $\alpha$-stable processes on $I R^{d}$ starting at 0 ; we assume that the processes are genuinely $d$-dimensional, that is, for each $j$, the distribution of $X_{j}(1)$ is not supported by a strict subspace of $I R^{d}$. We will suppose for simplicity that the processes $X_{j}(),. j=1,2, \ldots, N$ are i.i.d. but, as will be seen, this is not necessary. This implies for $\alpha \neq 1$ that for $t>0$, $E\left[e^{i<u, X_{j}(t)>}\right]=\exp \left(-|t| \int_{S_{d}}|<\theta, u>|^{\alpha}\left(1-i \operatorname{sign}(<\theta, u>) \tan \left(\frac{\pi \alpha}{2}\right)\right) \Gamma(d \theta)\right)$,

\footnotetext{
${ }^{1}$ Institut de Mathématiques, Ecole Polytechnique Fédérale de Lausanne, 1015 Lausanne, Switzerland. E-mail: thomas.mountford@epfl.ch
} 
while for $\alpha=1$,

$E\left[e^{i<u, X_{j}(t)>}\right]=\exp \left(-|t| \int_{S_{d}}|<\theta, u>|^{\alpha}\left(1+i \frac{2}{\pi} \operatorname{sign}(<\theta, u>) \operatorname{Ln}(|<\theta, u>|)\right) \Gamma(d \theta)\right)$,

where for each "direction" $1 \leqslant k \leqslant d, \int_{S_{d}} \theta_{k} \Gamma(d \theta)=0$ (see, e.g. Ref. 11). We suppose also that the processes satisfy Taylor's condition (A) (see the next section for a statement). This is automatic for $\alpha \geqslant 1$. We discuss loosening of these conditions at the end of the article. The reason for these assumptions is to profit from the work of Ref. 12 where these assumptions are in force.

For the moment we will restrict attention to one sided, onedimensional time processes $\left(X(t): t \geqslant 0\right.$ ) (as with the $X_{j}($.$) , we neglect$ the vector notation even though $X(t)$ may well be a multi-dimensional quantity in order to distinguish the one-dimensional time process from the multi-dimensional time process).

For these processes, for $t>0$ the sigma field $\mathcal{F}_{t}$ will represent the completion of the sigma field generated by $\{X(s): s \leqslant t\}$, in the multitime context we will use $\mathcal{F}_{t}$ for $\underline{t}=\left(t_{1}, t_{2}, \ldots, t_{N}\right)$ with $t_{i} \geqslant 0 \forall i$, the sigma field generated by $\left\{\bar{X}_{i}(s): s \leqslant t_{i}: i=1,2, \ldots, N\right\}$. We hope that context will be sufficiently strong to escape any confusion. The property that we will most often use is that for $0 \leqslant s<t$ the density of $X_{j}(t)-$ $X_{j}(s)$ is bounded by $\frac{K}{(t-s)^{d / \alpha}}$ for some constant, $K$, depending on the specific process but not on the particular $s$ and $t$. This bound follows from the boundedness of the density at time one and the scaling relations

$$
\forall c>0 \quad\left(c^{-1 / \alpha} X(c t):-\infty<t<\infty\right) \stackrel{D}{=}(X(t):-\infty<t<\infty)
$$

(see, e.g. Ref. 11).

A key element is the local time functional $L$ defined (at least) on intervals (or rectangles) $I=I_{1} \times I_{2} \times \cdots I_{N}\left(I_{i}\right.$ intervals in $I R$.), by

$$
L(I)=\lim _{\epsilon \rightarrow 0} \frac{1}{(2 \epsilon)^{d}} \lambda_{N}\left(\underline{t} \in I:|\underline{X}(\underline{t})|_{\infty}<\epsilon\right) .
$$

This local time will exist a.s. if and only if $N \geqslant d / \alpha$ (see, e.g. Ref. 4 or Theorem 1.1 of Ref. 5), indeed if $N<d / \alpha$, then a.s. $\underline{X}(\underline{t})$ will be nonzero for all nonzero $\underline{t}$ (see, e.g. Ref. 8 or Ref. 9).

For a set $E \subset I R^{N}$, and a continuous increasing function $\phi$ on the reals with $\phi(0)=0$, the measure $m_{\phi}\{E\}$ is defined as the (increasing) limit of $m_{\phi}^{\delta}\{E\}$ where the latter quantity is the infimum over all coverings of 
$E$ by cubes $\left\{J_{k}\right\}_{k} \geqslant 1$ such that for each $k,\left|J_{k}\right|$, the diameter of $J_{k}$, is less than $\delta$, of

$$
\sum_{k} \phi\left(\left|J_{k}\right|\right)
$$

(see, e.g. Ref. 2 or Ref. 10). We show

Theorem 1.1. For $\underline{X}$ as above with $N>d / \alpha$, there exists a finite, strictly positive constant $c$ so that a.s. $\forall$ finite interval $I=I_{1} \times I_{2} \cdots \times$ $I_{N} \subseteq I R^{N}$

$$
m_{\phi}\{\underline{t} \in I: \underline{X}(\underline{t})=0\}=c L(I),
$$

where $\phi(v)=v^{N-d / \alpha}\left(\log \log \left(\frac{1}{v}\right)\right)^{d / \alpha}$ and $L$ is the local time.

The result for the Brownian case has already been shown in Ref. 7 . As before this question is "half" solved by Xiao ${ }^{(12)}$ and Khoshenevisan et $a l$. ; $^{(4)}$ it was noted by Khoshenevisan et al. ${ }^{(4)}$ that the arguments of the former paper allied with its own results led to the conclusion that

$$
m_{\phi}\{\underline{t} \in I: \underline{X}(\underline{t})=0\}>0 \quad \text { a.s. if } L(I)>0 .
$$

Essentially our task reduces to proving a converse inequality

Proposition 1.2. $\exists$ nonrandom $K<\infty$ so that for any closed bounded interval $I$, not intersecting the axes, a.s.

$$
m_{\phi}\{t \in I: \underline{X}(t)=0\}<K L(I) .
$$

Given this result the arguments of Section 5 of Ref. 7 lead easily to Theorem 1.1 given the similarity of the approach we merely provide a sketch. One first notes that, given the continuity properties of the local times and Proposition 1.2

$$
m_{\phi}\{\underline{t} \in I: \underline{X}(\underline{t})=0\}=c L(I)
$$

will hold a.s. for all intervals $I$ if it holds for each interval a.s. We consider for concreteness the interval $I=[0,1]^{N}$. The function $f: I R^{d} \rightarrow I R_{+}$ is defined as

$$
E^{\underline{x}}\left[m_{\phi}\left\{\underline{t} \in[0,1]^{N}: \underline{X}(\underline{t})=0\right\}\right]=E\left[m_{\phi}\left\{\underline{t} \in[0,1]^{N}: \underline{X}(\underline{t})=0\right\} \mid \underline{X}(\underline{0})=\underline{x}\right] .
$$


This function is easily seen (via Proposition 1.2 and continuity properties of local times) to be continuous in $\underline{x}$ and integrable. Furthermore by scaling for any interval $\prod_{j}\left[\frac{i_{j}}{2^{n}}, \frac{i_{j}+1}{2^{n}}\right]$,

$$
E\left[m_{\phi}\left\{\underline{t} \in \prod_{j}\left[\frac{i_{j}}{2^{n}}, \frac{i_{j}+1}{2^{n}}\right]: \underline{X}(\underline{t})=0\right\} \mid \underline{X}\left(\frac{i_{1}}{2^{n}} \frac{i_{2}}{2^{n}} \cdots \frac{i_{N}}{2^{n}}\right)=\underline{x}\right]=f\left(\underline{x} 2^{n / \alpha}\right) 2^{-(N-d / \alpha) n} .
$$

Proof of Theorem 1.1 is concluded by showing that

$$
\sum_{0 \leqslant i_{1}, i_{2}, \ldots, i_{N} \leqslant 2^{N}-1} f\left(\underline{X}\left(\left(\frac{i_{1}}{2^{n}} \frac{i_{2}}{2^{n}} \cdots \frac{i_{N}}{2^{n}}\right)\right) 2^{n / \alpha}\right) 2^{-(N-d / \alpha) n} \rightarrow m_{\phi}\{\underline{t} \in I: \underline{X}(\underline{t})=0\}
$$

in probability and that

$$
\sum_{0 \leqslant i_{1}, i_{2}, \ldots, i_{N} \leqslant 2^{N}-1} f\left(\underline{X}\left(\left(\frac{i_{1}}{2^{n}} \frac{i_{2}}{2^{n}} \cdots \frac{i_{N}}{2^{n}}\right)\right) 2^{n / \alpha}\right) 2^{-(N-d / \alpha) n} \rightarrow \int f(\underline{x}) d \underline{x} L\left([0,1]^{N}\right)
$$

(again in probability).

The approach will be a modification of Ref. 7 to find a useful lower bound on the probability of large values of $L\left([0, t]^{N}\right)$ for real $t$ small starting from an initial value

$$
\|\underline{X}(0)\|_{\infty}<\frac{t^{1 / \alpha}}{\left(\log \log \left(\frac{1}{t}\right)\right)^{1 / \alpha}} .
$$

The key step will be, essentially, to condition on an event $A=A(N, t)$ so that $\left(\underline{X}(\underline{s}): \underline{s} \in[0, t]^{N}\right)$ conditioned on event $A$ is a new process

$$
\left(\underline{Y}^{A}(\underline{s}): \underline{s} \in[0, t]^{N}\right)
$$

and that for this process the local time $L^{Y}\left([0, t]^{N}\right)$ is, with "reasonable" probability, $h$, as $t \rightarrow 0$ at least $C_{A} t^{N}$, where $C_{A}$ is of the order $\frac{1}{t^{d / \alpha}}\left(\log \log \left(\frac{1}{t}\right)\right)^{d / \alpha}$. Hence for any $t$ small,

$$
P\left(L\left([0, t]^{N}\right) \geqslant C_{A} t^{N}\right) \geqslant h P(A(N, t)) .
$$

We have many available possibilites for the process $\underline{Y}^{A}($.$) . There is a trade$ off between naturalness of process $\left(\underline{Y}^{A}(\underline{s}): \underline{s} \in[0, t]^{\bar{N})}\right.$ and the ease of calculations; we have opted for a process where calculations are reasonable, though it is not Markov. Nonmarkovness notwithstanding, the resulting process will be "positive recurrent" in a simple way.

The paper is organized as follows. In Section 2, we consider nonMarkov but "positive recurrent" processes derived from our original (one- 
dimensional time) stable process via suppressing certain types of jump and augmenting (by a bounded factor) the rates of others. In the succeeding section, we exploit this process to gain lower bounds on large deviation probabilities for the multi-dimensional time stable processes. In the last section, we establish Proposition 1.2, closely following the approach of Ref. 7 and then discuss extensions of the approach.

We will make use of various vectorial conventions: as previously mentioned, in a multi-dimensional context, an interval will refer to a subset that is a Cartesian product of one dimensional intervals. We will write for vectors $\underline{t}$ and $\underline{s} \in I R^{N}$ that $\underline{t} \leqslant \underline{s}$ if for each $i \in\{1,2, \ldots, N\}, t_{i} \leqslant s_{i}$, equally $\underline{t}<\underline{s}$ is to be understood componentwise. For a vector $\underline{u}=\left(u_{1}, u_{2}, \ldots, u_{N}\right)$ and a constant $c, \underline{u}+c$ is simply the vector $\left(u_{1}+c, u_{2}+c, \ldots, u_{N}+c\right)$. For a set $A \subset I R^{r}$ for $r$ a positive integer and a real $c, c A$ will denote the set $\{c \underline{x}: \underline{x} \in A\}$. For $y, \underline{x} \in I R^{r},\langle\underline{x}, \underline{y}\rangle$ and $\underline{x} . y$ will both denote the usual scaler product. For $\underline{x}$ in Euclidean space, $B(\underline{x}, v)$ will signify the Euclidean ball of radius $v$ centred at $\underline{x}$. The indicator functions will be written as $I_{A}$ where $A$ may be either a random event or a logical condition. Equally if we consider points on a sphere $\underline{\theta}$, the ball on the sphere $B(\underline{\theta}, \epsilon)$ will simply denote those points on the sphere within Euclidean distance $\epsilon$ of $\underline{\theta}$. The constant $C_{d}$ will denote the volume of the unit ball in $I R^{d}$.

\section{THE POSITIVE RECURRENT PROCESS}

In this section, we consider various processes derived from onedimensional time indexed strictly stable processes conditioned to not make any jumps exceeding a certain threshold. The object is, ultimately, to construct a "positive recurrent" process $\left(Z^{v}(t): t \geqslant 0\right)$ "close" (in a sense that will be made clear) to the original stable process which will have spatial scale $v$ and temporal scale $v^{\alpha}$ in the sense that

(i) for all times $t \geqslant v^{\alpha}, Z^{v}(t)$ will be of "order" $v$;

(ii) $Z^{v}(t+s)-Z^{v}(t)$ will be of order $s^{1 / \alpha} \wedge v$.

This process will not be (quite) Markov.

We first consider $X^{v}($.) the process with jumps of magnitude greater than $v$ suppressed. Then by "adding in" (or even reinforcing) some suppressed jumps of order $v$, we obtain a centred Levy process $Y^{v}($.$) . This$ process is then modified to become the target process $Z^{v}($.).

For an $\alpha$ stable process on $I R^{d}$, the Lévy measure $\rho(d \underline{x}), \underline{x} \in I R^{d}$ is of the form (see Ref. 11)

$$
\rho(d \underline{x})=\frac{d R}{R^{\alpha+1}} \rho^{\prime}(d \underline{\theta})
$$


for $\rho^{\prime}$ a measure on the sphere of radius 1 and

$$
R, \underline{\theta} \text { is } \underline{x} \text { in polar co-ordinates. }
$$

We suppose that the $\alpha$-stable process in $I R^{d},(X(t): t \geqslant 0)$ satisfies Taylors condition (A): $p_{t}(x, y)>0$ for all strictly positive $t$ where $p_{t}(.,$.$) is the den-$ sity kernal for $X_{t}(\cdot)$.

We will at the end of the paper discuss how to extend our results to processes with drift.

Let $\left(X^{v}(t): t \geqslant 0\right)$ be the Lévy process with the same drift terms as $(X(t): t \geqslant 0)$ but with Lévy measure equal to

$$
\rho(d \underline{x}) I_{|\underline{x}| \leqslant v} .
$$

Equally one can describe $\left(X^{v}(t): t \geqslant 0\right)$ as the process obtained from $(X(t): t \geqslant 0)$ by suppressing all jumps of magnitude greater than $v$. We note here that $\left(X^{v}(s): 0 \leqslant s \leqslant t\right)$ can be simply seen as $(X(s): 0 \leqslant s \leqslant t)$ conditioned on an event of probability $e^{-k t / v^{\alpha}}$ where $k=\rho\{x:|x| \geqslant 1\}$ and so on time scales of the order $v^{\alpha}, X^{v}($.) will exhibit much the same behaviour as $X($.$) .$

While the expectation for $X(t)$ need not exist, that of $X^{v}(t)$ will certainly be defined and because of the stationary increments property and the stability it must be equal to $\underline{c} t v^{1-\alpha}$ where $\underline{c}$ is a fixed vector in $I R^{d}$. We now show that we can find a convenient Lévy process absolutely continuous with respect to $X($.$) of zero mean and with the size of all jumps$ bounded by $2 v$.

Proposition 2.1. There exists $M<\infty$ and $A \subset B(\underline{0}, 2) \backslash B(\underline{0}, 1)$ so that $\forall v>0$, the Lévy process $Y^{v}$ (.) with Lévy measure

$$
\rho^{Y}(d x)=\rho(d x) I_{|x| \leqslant v}+M \rho(d x) I_{v A}(x),
$$

satisfies

$$
E\left[Y^{v}(t)\right]=0 \quad \forall t>0 .
$$

It is only necessary to treat $v=1$ by scaling. The case $\alpha>1$ is easiest and we tackle it first. In this case $E[X(1)]=0$. Suppose that $E\left[X^{1}(1)\right] \neq 0$ (otherwise we may simply take $A$ to be the empty set). Then we choose $A$ to be $B(\underline{0}, 2) \backslash B(\underline{0}, 1)$ and $M$ to be $\int_{B(\underline{0}, 1)^{c}}|x| \rho(d x)$ divided by $\int_{B(\underline{0}, 2) \backslash B(\underline{0}, 1)}|x| \rho(d x)$.

We now suppose that $\alpha \leqslant 1$. In this case condition (A) and strict stability implies condition 
$\left(A^{\prime}\right)$ : the measure $\rho$ is not concentrated on any half space in $I R^{d}$.

In this case the proposition is a consequence of two lemmas.

Lemma 2.2. Under condition $\left(A^{\prime}\right)$ there exists an $\epsilon>0$ so that for every direction $\underline{\theta}$ on the unit sphere $S^{d}, \rho^{\prime}(\gamma:\langle\underline{\theta}, \gamma\rangle<-2 \epsilon) \geqslant$ $2 \epsilon$ and consequently $\exists \epsilon>0, \delta>0$ so that $\forall \underline{\theta} \in S^{d}, \quad \exists \gamma:<\gamma, \underline{\theta}>\leqslant$ $-\frac{3}{2} \epsilon$ so that $\rho^{\prime}(B(\gamma, \epsilon))>\delta$.

Proof. The first part is simply a compactness argument: suppose not then $\forall n \exists \underline{\theta}_{n} \in S_{d}$ so that the condition fails with $\epsilon=1 / n$. By compactness of $S_{d}, \exists$ a subsequence $\underline{\theta}_{n_{k}} \rightarrow \underline{\theta}_{0}$. Then the condition $\left(A^{\prime}\right)$ is violated by the half space $\left\{x:\left\langle x, \underline{\theta}_{0}\right\rangle \geqslant 0\right\}$. Given such an $\epsilon>0$ and any $\underline{\theta} \epsilon$ $S_{d}$, we can cover the set $\{\gamma:<\gamma, \underline{\theta}>\leqslant-2 \epsilon\}$ with a finite number, $M=M_{\theta}$, of $\epsilon$-balls, $B\left(\gamma_{1}, \epsilon\right), \ldots, B\left(\gamma_{i}, \epsilon\right), \ldots, B\left(\gamma_{M}, \epsilon\right)$ where for each $i, \gamma_{i} \in\{\gamma:<$ $\gamma, \underline{\theta}>\leqslant-2 \epsilon\}$. This cover must also cover $\left\{\gamma:<\gamma, \theta^{\prime}>\leqslant-\frac{3}{2} \epsilon\right\}$ for all $\underline{\theta}^{\prime}$ in a neighbourhood, $C_{\theta}$, of $\underline{\theta}$ and we may also take $C_{\theta}$ so that for each $i, \gamma_{i} \in\left\{\gamma:<\gamma, \underline{\theta}^{\prime}>\leqslant-\frac{3}{2} \epsilon\right\}$ for all $\theta^{\prime} \in C_{\underline{\theta}}$. Again we take a finite collection $C_{\theta_{1}}, C_{\theta_{2}}, \ldots, C_{\theta_{n}}$ covering the sphere and let $M=\max _{1 \leqslant i \leqslant n}\left\{M_{\theta_{i}}\right\}$, then for any $\theta$ we have $\gamma \in\left\{\gamma^{\prime}:<\gamma^{\prime}, \theta>\leqslant-\frac{3}{2} \epsilon\right\}$ so that

$$
\rho^{\prime}(B(\gamma, \epsilon)) \geqslant \epsilon / M \text {. }
$$

The result now follows by reducing $\epsilon$.

Lemma 2.3. Under condition $\left(A^{\prime}\right)$, for some $\epsilon>0$, there exist disjoint $B\left(\theta_{1}, \underline{\epsilon}\right), B\left(\underline{\theta}_{2}, \epsilon\right), \ldots, B\left(\underline{\theta}_{d}, \epsilon\right)$ so that $\underline{x}_{i}=\int_{B\left(\theta_{i}, \epsilon\right)} \rho^{\prime}(d \underline{\theta}) \underline{\theta}$ are linearily independent.

Proof. Let $\epsilon, \delta$ be fixed as in Lemma 2.2 and such that the previous lemma holds for $K \epsilon, K^{d+2} \delta$ where $K$ is large but fixed. We choose $\underline{\theta}_{1}, \ldots, \underline{\theta}_{d}$ in succession (and so $\underline{x}_{1}, \ldots, \underline{x}_{d}$ where $\underline{x}_{i}=\int_{B\left(\underline{\theta}_{i}, \epsilon\right)} \underline{\theta} \rho^{\prime}(d \underline{\theta})$ ).

First $\underline{\theta}_{1}$ is chosen so that $\rho^{\prime}\left(B\left(\underline{\theta}_{1}, \epsilon\right)\right)>\delta$ (which is possible if $K$ is large).

Now, having chosen the first $k$ points $\underline{\theta}_{1}, \ldots, \underline{\theta}_{k}$, let $A$ be the subspace generated by $\underline{x}_{1}, \underline{x}_{2}, \ldots, \underline{x}_{k}$ and $V$ a halfspace whose boundary contains $A$. By Lemma 2.2 there is $\underline{\theta}_{k+1}$ so that

$$
\begin{gathered}
\rho^{\prime}\left(B\left(\underline{\theta}_{k+1}, \epsilon\right)\right)>\delta, \\
B\left(\underline{\theta}_{k+1}, \epsilon\right) \cap B\left(\underline{\theta}_{i}, \epsilon\right) \neq \emptyset \quad i=1,2, \ldots, k
\end{gathered}
$$


and so that there exists $\underline{\Theta}:<\underline{\Theta}, \underline{x}_{i}>=0 i=1,2, \ldots, k$ and $<\underline{\Theta}, \underline{\theta^{\prime}}>\leqslant$ $-\frac{K}{2} \epsilon \forall \quad \underline{\theta}^{\prime} \in B\left(\underline{\theta}_{k+1}, \epsilon\right)$. This implies that

$$
\underline{x}_{k+1}=\int_{B\left(\theta_{k+1}, \epsilon\right)} \rho^{\prime}(d \theta) \underline{\theta}
$$

is not a linear combination of $\underline{x}, \ldots, \underline{x}_{k}$.

Continuing we obtain $\underline{\theta}_{1}, \underline{\theta}_{2}, \ldots \underline{\theta}_{d}, \underline{x}_{1}, \underline{x}_{2}, \ldots, \underline{x}_{d}$.

We now return to Proposition 2.1 for the case $\alpha \leqslant 1$, which is now a simple consequence of these two lemmas.

Proof. Suppose without loss of generality that $E\left[X^{1}(1)\right]=\underline{x}_{0} \neq \underline{0}$. Let $K$ be the set of points $\underline{x} \in I R^{d}$ of the form

$$
\underline{x}=\sum_{j=1}^{R} c_{j} \int_{A_{j}} \underline{\theta} \rho^{\prime}(d \underline{\theta}),
$$

where the $A_{j}$ are Borel subsets of $S^{d}$, the $c_{j}$ are strictly positive and the vectors $\int_{A_{j}} \underline{\theta} \rho^{\prime}(d \underline{\theta})$ span $I R^{d}$. Clearly $K$ is an open cone in $I R^{d}$. Now note that, given the decomposition of the Levy measure $\rho$ via $\rho^{\prime}$, if $-\underline{x}_{0} \in K$, then we can find $M>0$ and Borel subset $H_{x_{0}}$ of $B(\underline{0}, 2) \backslash B(\underline{0}, 1)$ so that

$$
-\underline{x}_{0}=M \int_{H_{x_{0}}} u \rho(d u) .
$$

Thus the proposition will quickly follow if we can show that $K$ is equal to $I R^{d}$, which will certainly follow if $\underline{0} \in K$. Since $K$ is an open cone the alternative is that $\underline{0}$ is on the boundary of $K$. But by the separation theorem ( $K$ is convex) there would then exist $\underline{v} \in I R^{d}$ so that $K \subset\{\underline{u}:<\underline{u}, \underline{v}>>0\}$. A contradiction quickly arrives as, by the condition $\left(A^{\prime}\right)$, measure $\rho^{\prime}$ charges the unit sphere intersected with $\{\underline{u}:<\underline{u}, \underline{v}><0\}$.

Having defined $Y^{v}$ (.) we take $M$ to be as given in Proposition 2.1. The Lévy process $Y^{v}($.$) is, according to dimension d$ and index $\alpha$, transient or null recurrent: it cannot be positive recurrent. We now proceed to modify this process so that while it loses the Markov property, it is "positive recurrent". We choose $M_{1}>>1$ but not depending on $v$ and we define process $\left(Z^{v}(t): t \geqslant 0\right)$ so that over time intervals $\left[i v^{\alpha},\left(i+\frac{1}{2}\right) v^{\alpha}\right)$ it evolves as the Lévy process $Y^{v}($.$) , while over the interval \left[\left(i+\frac{1}{2}\right) v^{\alpha},(i+1) v^{\alpha}\right)$ by the rule:

(a) if $\left|Z^{v}\left(i v^{\alpha}\right)\right| \leqslant M_{1} v$, then on the interval $\left[\left(i+\frac{1}{2}\right) v^{\alpha},(i+1) v^{\alpha}\right)$, $Z^{v}$ (.) evolves as the process $\left(Y^{v}(t): t \geqslant 0\right)$, but 
(b) if $\left|Z^{v}\left(i v^{\alpha}\right)\right|>M_{1} v$, then on the interval $\left[\left(i+\frac{1}{2}\right) v^{\alpha},(i+1) v^{\alpha}\right)$, $Z^{v}\left(\right.$.) evolves as the Levy process $Y^{v}$ (.) except that the jumps of size $\frac{1}{2} v<|y|<2 v$ for $y$ with $<y, Z^{v}\left(i v^{\alpha}\right)>>0$ are suppressed and jumps in $2 v<|y|<4 v$ with $<y, Z^{v}\left(i v^{\alpha}\right)><0$ are unsuppressed.

Even though the process $Z^{v}$ (.) is not Markov, it evolves as a Lévy process on each interval $\left[i v^{\alpha},\left(i+\frac{1}{2}\right) v^{\alpha}\right]$ and on each interval $[(i+$ $\left.\left.\frac{1}{2}\right) v^{\alpha},(i+1) v^{\alpha}\right]$ (though which law depends on the value $Z^{v}\left(i v^{\alpha}\right)$ ) and so we have easily

Lemma 2.4. Given $0 \leqslant s<t$, there exists a constant, $c_{1}$ (not depending on $v$ ) so that the conditional density of $Z^{v}(t)$ given $\mathcal{F}_{s}=$ $\sigma\left\{Z^{v}(u): u \leqslant s\right\}$ is bounded by the maximum of $c_{1} / v^{d}$ and $c_{1} /(t-s)^{d / \alpha}$.

Proof. We treat first the case $t-s \leqslant v^{\alpha} / 2$. In this case the conditional distribution of $Z^{v}(t)-Z^{v}(s)$ given $\mathcal{F}_{s}$ can be written as $Z_{1}+Z_{2}$ where the $Z_{i}$ are conditionally independent and $Z_{1}$ is equal in distribution to $X^{\frac{v}{2}}(t-s) . X^{\frac{v}{2}}(t-s)$ is the random variable $X(t-s)$ conditioned on an event (no "large" jumps) of probability bounded away from zero. Accordingly the density of $Z_{1}$ is bounded by $c /(t-s)^{d / \alpha}$ for some $c$ not depending on $v, t$ or $s$. So the density of $Z_{1}+Z_{2}$ is also so bounded. The general case now follows as the $L_{\infty}$ bound of the density of $Z^{v}(t)$ given $\mathcal{F}_{s}=\sigma\left\{Z^{v}(u): u \leqslant s\right\}$ is less than that for the density of $Z^{v}(t)$ given $\mathcal{F}_{t-v^{\alpha} / 2}$.

Lemma 2.5. For each $L>0$, there exists a strictly positive $d_{L}$ so that uniformly on $|x| \leqslant L$, starting from $x$ at time 0 , the conditional density of $Z^{v}\left(v^{\alpha}\right)$ is at least $d_{L} / v^{d}$ at each point within $d_{L}$ of the origin.

Proof. We need only consider the case $v=1$ by scaling. We may consider instead of the process $Z^{1}($.$) , the Lévy process \left(X^{1 / 2}(s): 0 \leqslant\right.$ $s \leqslant 1)$ since this process arises from the other by conditioning on an event of probability bounded away from zero, namely the non occurrence of any jumps of size greater than one half on the unit time interval.

We first consider for this process, starting at zero, the density at position $y$ at time $c<1, p^{\prime}(c, y)$. By elementary Fourier analysis this is 
equal to

$$
\begin{gathered}
\frac{1}{(2 \pi)^{d}} \int_{I R^{d}} e^{-i u \cdot y} E\left[e^{i u \cdot X^{1 / 2}(c)}\right] d u \\
=\frac{1}{(2 \pi)^{d}} \int_{I R^{d}} e^{-i u \cdot y} E\left[e^{i u \cdot X(c)}\right] d u+\frac{1}{(2 \pi)^{d}} \int_{I R^{d}} e^{-i u \cdot y}\left(E\left[e^{i u \cdot X^{1 / 2}(c)}\right]-E\left[e^{i u \cdot X(c)}\right]\right) d u \\
=p^{X}(c, y)+o\left(c^{d / \alpha}\right),
\end{gathered}
$$

where $p^{X}(c,$.$) is the density of X(c)$ conditioned on starting from the origin. Since $p^{X}(c, y)=c^{-d / \alpha} p^{X}\left(1, y / c^{1 / \alpha}\right)$ and $p^{X}(1,$.$) is a continuous$ function, we conclude that for $c$ small enough $p^{\prime}(c, y)>1$ for all $|y| \ll c^{1 / \alpha}$. Fix such a $c$.

For fixed $x$, it is easily seen, there is a strictly positive probability, $f(x)$ that, starting from $x, X^{1 / 2}(1-c)$ has magnitude less than $c^{1 / \alpha} / 4$. Thus by compactness of the ball of radius $L$ there is a strictly positive $f$ so that uniformly over $|x| \leqslant L, \quad P^{x}\left(\left|X^{1 / 2}(1-c)\right| \leqslant c^{1 / \alpha} / 2\right.$. From this and the Markov property at time $1-c$ we have that uniformly over $|x| \leqslant L$ the density at $y$ is at least $f$ for all $y$ of magnitude less than $c^{1 / \alpha} / 2$.

It is easy but laborious to show

Proposition 2.6. There exists $M_{2}>M_{1}$ and $\delta, c>0$ not depending on $v$ so that if

$$
\left|Z^{v}(0)\right|>M_{2} v,
$$

then for $\sigma=\inf \left\{i v^{\alpha}:\left|Z^{v}\left(i v^{\alpha}\right)\right|<M_{2} v\right\}$,

$$
W_{n}=e^{c\left|Z^{v}\left(n v^{\alpha} \wedge \sigma\right)\right|+\delta\left(n v^{\alpha} \wedge \sigma\right)}
$$

is a supermartingale.

Corollary 2.7. The discrete time Markov chain $\left(Z^{v}\left(n v^{\alpha}\right): n \geqslant 0\right)$ has a unique stationary distribution.

Proof. We define the stopping times (with respect to the continuous time filtration)

$$
\begin{aligned}
& \sigma_{0}=\inf \left\{t>0:\left|Z^{v}(t)\right|>M_{2} v\right\} \text { and for } i \geqslant 0, \quad \sigma_{i}^{\prime}=\inf \left\{n v^{\alpha}>\sigma_{i}\right\}, \\
& \tau_{i}=\inf \left\{n v^{\alpha} \geqslant \sigma_{i}^{\prime}:\left|Z^{v}\left(n v^{\alpha}\right)\right|<M_{2} v\right\}, \\
& \sigma_{i+1}=\inf \left\{t>\tau_{i}:\left|Z^{v}(t)\right|>M_{2} v\right\} . \text { The } \tau_{i}, \sigma_{i}^{\prime} \text { are not stopping times with }
\end{aligned}
$$
respect to the the natural filtration of $\left\{Z^{v}\left(n v^{\alpha}\right): n \geqslant 0\right\}$ but this will not affect things.

Since the jumps for the continuous time process $\left\{Z^{v}(t): t \geqslant 0\right\}$ are bounded by $4 v$ in magnitude, it is immediate that (with the possible exception of $\left.\sigma_{0}\right)\left|Z^{v}\left(\sigma_{i}\right)\right| \leqslant\left(M_{2}+4\right) v \forall i$. From this and the Markov property 
(given $\mathcal{F}_{j v^{\alpha}}$ ) on intervals $\left[j v^{\alpha},(j+1) v^{\alpha}\right]$, we have (again with the possible exception of $\sigma_{0}$ ) that there exists non random $K$ so that for all $i$ and $v$,

$$
E\left[e^{\delta\left|Z^{v}\left(\sigma_{i}^{\prime}\right)\right|} \mid \mathcal{F}_{\sigma_{i}}\right] \leqslant K .
$$

This and Proposition 2.6 ensure that

$$
E\left[e^{\delta\left(\tau_{i}-\sigma_{i}^{\prime}\right)} \mid \mathcal{F}_{\sigma_{i}}\right] \leqslant K^{\prime} .
$$

From this we easily deduce that for all initial values $z \in I R^{d}$, the distributions

$$
\mu_{n}(A)=\frac{1}{n} \sum_{i=1}^{n} P^{z}\left(Z^{v}\left(i v^{\alpha}\right) \in A\right),
$$

satisfy

$\exists K_{1}, K_{2}<\infty, c>0 \quad$ so that for all $x \limsup _{n \rightarrow \infty} \mu_{n}\left(B(0, x v)^{c}\right) \leqslant K_{1} e^{-K_{2} x}$.

This inequality firstly implies that the measures $\left\{\mu_{n}\right\}_{n} \geqslant 1$ are tight and thus (see, e.g. Ref. 1) possess a convergent subsequence in the weak topology. Any limit is necessarily a stationary distribution for the semigroup $P$ (see, e.g. Ref. 6, Proposition 1.8) and will inherit the exponetial bound on tail probabilities. This ensures that a stationary distribution, $\mu^{v}$, for the discrete time process exists and that it satisfies $\mu^{v}\left(B(0, v x)^{c}\right) \leqslant K_{1} e^{-K_{2} x}$ for all positive $x$.

To establish uniqueness of the stationary measure, note first that by Lemma 2.5, any two realisations $Z^{v}\left(\right.$.) and $Z^{v^{\prime}}($.) may be coupled together so that after first meeting (at a time $i v^{\alpha}$ ) they are equal thereafter and so that for $L>0$ fixed and all positive integer $n$,

$$
P\left(Z^{v}\left(n v^{\alpha}\right)=Z^{v \prime}\left(n v^{\alpha}\right) \mid Z^{v}\left(m v^{\alpha}\right), Z^{v \prime}\left(m v^{\alpha}\right) \quad 0 \leqslant m<n\right)>d_{L} C_{d}\left(d_{L}\right)^{d}>0,
$$

if both $Z^{v}\left((n-1) v^{\alpha}\right)$ and $Z^{v \prime}\left((n-1) v^{\alpha}\right)$ are less than $L v$ in magnitude.

By our exponential bounds we have that if $L$ is fixed sufficiently large then with probability tending to one as $n$ tends to infinity,

$$
\frac{\# i<n:\left|Z^{v}\left(i v^{\alpha}\right)\right|<L}{n}>\frac{3}{4}
$$

and likewise for $Z^{v \prime}($.$) . Thus with probability tending to one as n$ tends to infinity

$$
\frac{\# i<n:\left|Z^{v}\left(i v^{\alpha}\right)\right|,\left|Z^{v^{\prime}}\left(i v^{\alpha}\right)\right|<L}{n}>\frac{1}{2} .
$$

This implies that for the above coupling with probability tending to one as $n$ tends to infinity, $Z^{v}\left(n v^{\alpha}\right)=Z^{v \prime}\left(n v^{\alpha}\right)$. By standard arguments this yields the desired unicity. 
We are ultimately interested in the behaviour of the continuous time process

As noted, the process $\left(Z^{v}(t): t \geqslant 0\right)$ is non-Markov. However given stationary measure $\mu^{v}$ for the discrete time process, we call $\pi^{v, c}$ the stationary measure for the continuous time process $\left(Z^{v}(t): t \geqslant 0\right)$, where

$$
\pi^{v, c}(A)=\frac{1}{v^{\alpha}} \int_{0}^{v^{\alpha}} P^{\mu^{v}}\left(Z^{v}(s) \in A\right) d s .
$$

We now assemble some elementary properties of this stationary distribution. The following comes directly from the results for $\mu^{v}$ and Lemmas 2.4 and 2.5 .

Proposition 2.8. The "stationary" probability $\pi^{v, c}$ for the process $\left(Z^{v}(t): t \geqslant 0\right)$ is absolutely continuous with respect to Lebesgue measure with density bounded by $c_{2} / v^{d}$ and greater than $c_{3} / v^{d}$ in a neighbourhood of the origin containing $B\left(0, v c_{4}\right)$ for some strictly positive $c_{4}$ not depending on $v$.

The results and arguments given above enable one to conclude

Proposition 2.9. There are strictly positive $H(>1)$ and $c_{5}$ so that for the continuous time process $\left(Z^{v}(t): t \geqslant 0\right)$ :

(i) irrespective of $t>s \geqslant 0$ and $Z^{v}(0)=y \in I R^{d}$, the conditional density of $Z^{v}(t)$ given $\left\{Z^{v}(r): r \leqslant s\right\}$ is bounded by $\frac{1}{c_{5}}\left(\frac{1}{v^{d}} \vee \frac{1}{(t-s)^{d / \alpha}}\right)$;

(ii) for all $t>H v^{\alpha}$, and all $Z^{v}(0)$ of magnitude less than $v$, the conditional density of $Z^{v}(t)$ in $B\left(0, c_{5} v\right)$ is bounded below by $c_{5} / v^{d}$.

Given this we can establish the key bounds for the local times at zero of the multiparameter process $\underline{X}$.

Proposition 2.10. For process $\underline{Z}^{v}\left(t_{1}, \ldots, t_{N}\right)=\sum_{j=1}^{N} Z_{j}^{v}\left(t_{j}\right)$, where $Z_{j}^{v}$ are independent Markov processes with the same semigroup as $Z^{v}$ (.) above and with $\left|Z_{j}^{v}(0)\right| \leqslant v \forall j$

(i) there exists strictly positive $c_{6}$ not depending on $v$ so that for all $t \geqslant 2 H v^{\alpha}$ (H the constant of Proposition 2.9), $E\left[L\left(\left[\frac{t}{2}, t\right]^{N}\right)\right] \geqslant$ $c_{6} t^{N} / v^{d}$

(ii) there exists finite positive $c_{7}$ not depending on $v$ so that for all $t \geqslant 2 H v^{\alpha}(H$ the constant of Proposition 2.9$\left.), E\left[\left(L\left(\left[\frac{t}{2}, t\right]^{N}\right)\right]\right)^{2}\right] \leqslant$ $c_{7} t^{2 N} / v^{2 d}$. 
Proof. For $\left(s_{1}, s_{2}, \ldots, s_{N}\right) \in\left[\frac{t}{2}, t\right]^{N}$ with $s_{i} \geqslant H v^{\alpha} \forall i$, we have that $Z^{v}\left(s_{1}, \ldots, s_{N}\right)$ has density at zero at least $c_{5}^{(d+1)(N-1)}\left(\frac{\left(C_{d}\right)^{N-1}}{N^{(N-1) d}}\right) \frac{c_{5}}{v^{d}}$ where, recall, $C_{d}$ is the volume of a radius one sphere in $d$ dimensions. So, see Ref. 3,

$$
E\left[L\left(\left[\frac{t}{2}, t\right]^{N}\right)\right] \geqslant c_{6} t^{N} / v^{d}
$$

with $c_{6}=c_{5}^{(d+1)(N-1)}\left(\frac{\left(C_{d}\right)^{N-1}}{N(N-1) d}\right) c_{5} / 2^{N}$.

For the second bound we have (see, e.g. Ref. 3), that

$$
E\left[\left(L\left(\left[\frac{t}{2}, t\right]^{N}\right)\right)^{2}\right]=\int_{\left[\frac{t}{2}, t\right]^{N}} \int_{\left[\frac{t}{2}, t\right]^{N}} d \underline{s} d \underline{s}^{\prime} p\left(\underline{0}, \underline{0}, \underline{s}, \underline{s}^{\prime}\right),
$$

where $p\left(\underline{x}, \underline{y}, \underline{s}, \underline{s}^{\prime}\right)$ is the joint density of $\underline{Z}^{v}(\underline{s})$ and $\underline{Z}^{v}\left(\underline{s}^{\prime}\right)$ at $(\underline{x}, y)$. Now for given $\underline{s}$ and $\underline{s}^{\prime}$, let index $i$ be such that $\left|s_{i}-s_{i}^{\prime}\right|=\sup _{1 \leqslant j \leqslant n}\left|s_{j}-s_{j}^{\prime}\right|$. Without loss of generality suppose that $\left|s_{i}-s_{i}^{\prime}\right|=s_{i}-s_{i}^{\prime}$. Then applying Lemma 2.4 twice and using the fact that the density of a convolution is bounded by the smallest $L^{\infty}$ bound for the densities of the individual random variables, we find that

$$
p\left(\underline{0}, \underline{0}, \underline{s}, \underline{s}^{\prime}\right) \leqslant \frac{1}{c_{5}^{2}} \frac{1}{v^{d}}\left(\frac{1}{v^{d}} \wedge \frac{1}{\left|s_{i}-s_{i}^{\prime}\right|^{d / \alpha}}\right) .
$$

Integrating this over $\left[\frac{t}{2}, t\right]^{N} \times\left[\frac{t}{2}, t\right]^{N}$ gives the second moment bound.

This gives the immediate corollary

Corollary 2.11. For $\underline{Z}^{v}($.$) as in Proposition 2.10, above, so that$ $\left|Z_{j}^{v}().\right| \leqslant v \forall 1 \leqslant j \leqslant N$, there exists a strictly positive constant $c_{g}$ not depending on the initial values of the $Z_{j}^{v}(),$.$v or t$ so that for all $v$ and all $\left.t \geqslant 2 H v^{\alpha}, P\left(L\left(\left[\frac{t}{2}, t\right]^{N}\right)\right] \geqslant c_{g} t^{N} / v^{d}\right) \geqslant c_{g}$.

\section{APPLICATION TO THE ORIGINAL PROCESS}

We now consider using the results of the preceding section to gain information about the original process $X$ and $\underline{X}$. As already noted the process $\left(Z^{v}(t): t \geqslant 0\right)$ is simply the original process $(X(t): t \geqslant 0)$ conditioned on not making certain jumps over given intervals and having certain jump rates enhanced.

Proposition 3.1. Suppose that $\left|\sum_{j=1}^{N} X_{j}(0)\right| \leqslant N v$ and that $t / v^{\alpha}>$ $2 H$ for $H$ the constant of Proposition 2.9, then for $c_{8} \in(0, \infty)$ not depend- 
ing on $t$ or $v$,

$$
P\left[L\left(\left[\frac{t}{2}, t\right]^{N}\right)>c_{g} t^{N} / v^{d}\right]>c_{g} e^{-c_{8} t / v^{\alpha}},
$$

where $c_{g}$ is the constant of Proposition 2.9.

Proof. By linearity of $\underline{X}$ and the stationary independent increments property of Lévy processes we may suppose that for $j=1,2, \ldots, N$, $\left|X_{j}(0)\right| \leqslant v$.

We will take, without loss of generality, $t$ to be an integer multiple of $v^{\alpha}$. Let $t=n v^{\alpha}$. The idea is to exploit Corollary 2.11, which gives information on our "artificial" process $\underline{Z}^{v}($.$) , to gain suitable lower bounds on$ probabilities for our process $\underline{X}$. We first establish some new notation while recalling how (one- dimensional time process) $Z^{v}($.$) is derived from the$ original (one-dimensional time process) $X($.$) by suppressing some jumps$ and by increasing by a (bounded) factor the jump rates for other types of jumps (all drift term are retained):

(i) all jumps of magnitude greater than $4 v$ are suppressed.

(ii) no jump of magnitude less than $\frac{1}{2} v$ is suppressed.

Furthermore for $s \in\left[i v^{\alpha},\left(i+\frac{1}{2}\right) v^{\alpha}\right)$ the set $\left\{y: \frac{1}{2} v \leqslant|y| \leqslant 4 v\right\}$ is partitioned into three subsets:

$J_{i}^{1}$ for which the jump rate is zero,

$J_{i}^{2}$ on which the jump rate corresponds to $\rho$ the Levy measure of process $X($.$) ,$

$J_{i}^{3}$ on which the jump rate corresponds to $M \rho$.

Likewise on $s \in\left[i v^{\alpha},\left(i+\frac{1}{2}\right) v^{\alpha}\right)$ the set $\left\{y: \frac{1}{2} v \leqslant|y| \leqslant 4 v\right\}$ is partitioned into three subsets:

$H_{i}^{1}$ for which the jump rate is zero,

$H_{i}^{2}$ on which the jump rate corresponds to $\rho$ the Levy measure of process $X($.$) ,$

$H_{i}^{3}$ on which the jump rate corresponds to $M \rho$.

For clarity we will work on canonical space $(\Omega, \mathcal{F}, P)$ with $\Omega$ the space of cadlag functions $\omega: I R_{+}^{N} \rightarrow I R^{d}$, starting from $\left|\omega_{i}(0)\right| \leqslant v \forall 1 \leqslant$ $i \leqslant d$, with $\mathcal{F}$ the usual Borel sigmafield corresponding to Skorohod topology and $P$ the probability according to which the process $(w(s): s \geqslant 0)$ has the law of $\underline{X}($.$) . We let P_{2}$ be the probability on $(\Omega, \mathcal{F})$ under which $(w(s): s \geqslant 0)$ has the law of $Z^{v}($.$) and we take P_{1}$ to be the probability on this space corresponding to the "piecewise" Levy process which on interval 
$\left[i v^{\alpha},\left(i+\frac{1}{2}\right) v^{\alpha}\right)$ evolves like evolves like $Z^{v}\left(\right.$.) except that for jumps in $J_{i}^{3}$ the rates are given by $\rho$ and not $M \rho$ and likewise on $\left[\left(i+\frac{1}{2}\right) v^{\alpha},(i+1) v^{\alpha}\right)$ the jump rates for $H_{i}^{3}$ are those of $X($.). In words the jump rates are derived from those of $X($.) by making the same suppression as are made to get $Z^{v}($.) but without augmenting any rates.

We wish to prove a lower bound for $P(A)$ where $A$ is the set of paths $\left\{\omega: L\left(\omega,\left[\frac{t}{2}, t\right]^{N}\right)>c_{g} t^{N} / v^{d / \alpha}\right\}$. We first observe that

$$
P(A) \geqslant P(A \cap B)=P(B) P_{1}(A),
$$

where $B$ is the event that for all components $1 \leqslant j \leqslant N$, there are no jumps of magnitude greater than $4 v^{\alpha}$ and on time interval $\left[i v^{\alpha},\left(i+\frac{1}{2}\right) v^{\alpha}\right)$ no jumps in $J_{i}^{1} \quad 0 \leqslant i \leqslant n-1$ and on time interval $\left[\left(i+\frac{1}{2}\right) v^{\alpha},(i+1) v^{\alpha}\right)$ no jumps in $H_{i}^{1} 0 \leqslant i \leqslant n-1$. This easily leads to the bound

$$
P(A) \geqslant e^{-c_{8}^{\prime} n} P_{1}(A)
$$

for some constant $c_{8}^{\prime}$ depending only on the original process and not on $n$ or $v$. This is because the Levy measure of the "suppressed" zone is always bounded by a constant times $v^{\alpha}$. Thus to finish the proof of the lemma it will suffice to achieve a suitable lower bound for $P_{1}(A)$.

Since $P_{1}$ is absolutely continuous with respect to $P_{2}$, we have

$$
P_{1}(A)=\int_{A} \frac{d P_{1}}{d P_{2}}(\omega) d P_{2}(\omega) .
$$

Direct calculation gives that $\frac{d P_{1}}{d P_{2}}(\omega)$ is equal to $M^{-N(\omega)} e^{M-1} \geqslant M^{-N(\omega)}$ where

$$
\begin{aligned}
N(\omega)= & \sum_{i=0}^{n-1} \text { number of jumps in } J_{i}^{3} \text { during }\left[i v^{\alpha},\left(i+\frac{1}{2}\right) v^{\alpha}\right) \\
& +\sum_{i=0}^{n-1} \text { number of jumps in } H_{i}^{3} \text { during }\left[\left(i+\frac{1}{2}\right) v^{\alpha},(i+1) v^{\alpha}\right) .
\end{aligned}
$$

The random variable $N(\omega)$ is a Poisson process of random rate bounded by a constant $c_{9}$ times $v^{\alpha}$ where $c_{9}$ does not depend on $n$ or $v$. Hence there exists constant $c_{10}$ so that for all $n \geqslant 1$,

$$
P_{2}\left(N(\omega)>c_{10} n\right) \leqslant c_{g} / 2,
$$

where $c_{g}$ is the constant of Corollary 2.11. Thus we have by Corollary 2.11,

$$
P_{1}(A) \geqslant \int_{A \cap C} \frac{d P_{1}}{d P_{2}}(\omega) d P_{2}(\omega) \geqslant M^{-c_{10} n} c_{g} / 2 .
$$


where $C$ is the event $\left\{N(\omega) \leqslant c_{10} n\right\}$. Thus we have

$$
P(A) \geqslant e^{-c_{8}^{\prime} n} M^{-c_{10} n} c_{g} / 2 .
$$

We need now to transform this into a large deviation bound on the large deviations for multiparameter stable processes. The following lemma is elementary:

Lemma 3.2. For $(X(t): t \geqslant 0)$, with $|X(0)| \leqslant n^{1 /(2 d)} 2^{-n / \alpha}$, the probability that there exists a negative integer $i$ with $2^{i} \in\left[n^{2} 2^{-n}, 2^{-n / 2}\right]$ and so that $\left|X\left(2^{i}\right)\right| \geqslant n^{2 / \alpha} 2^{i / \alpha}$ is bounded by $c_{11} / n$ where $c_{11}$ is a finite constant not depending on $n$.

Let us take, for $\underline{t}>0$, the event $A(t, n)$ to be that there exists $j \in$ $\{1,2, \ldots, N\}$ and a negative integer $i$ with $2^{i} \in\left[n^{2} 2^{-n}, 2^{-n / 2}\right]$ so that $\left|X_{j}\left(t_{j}+2^{i}\right)-X_{j}\left(t_{j}\right)\right| \geqslant n^{2 / \alpha} 2^{i / \alpha}$, then we have

Corollary 3.3. For $\underline{t}>0$ the event $A(t, n)$ has probability bounded by $c_{12} / n$ for some $c_{12}$ depending on $N, \alpha$ but not on $n$ or $\underline{t}$. Furthermore if $\max _{i} t_{i}>c>0$ then the probability of the event $A(t, n) \cap\{|\underline{X}(t)| \leqslant$ $\left.n^{1 / 2 d} 2^{-n / \alpha}\right\}$ is less than $c_{13} 2^{-d n / \alpha} / n^{1 / 2}$ where $c_{13}=c_{13}(c)$ depends on $c$ but not on $n$ or $t$.

We can now give a bound on aberrant LIL behaviour for the local time around a time point $\underline{t}>0$. Define the event $C(n, \underline{t})$ (for such $\underline{t}$ ) to be $\left\{|\underline{X}(t)| \leqslant n^{1 / 2 d} 2^{-n / \alpha}\right\} \cap\left\{\nexists s \in\left[n^{2} 2^{-n}, 2^{-n / 2}\right]: L([t, \underline{t}+s]) \geqslant h s^{N-d / \alpha} \log (n)^{d / \alpha}\right\}$ $\backslash A(\underline{t}, n)$. Note we have not yet fully specfied the event $C(n, \underline{t})$ as the constant $h$ is not fixed. We will later fix it small but strictly positive in a way not depending on $n$.

Proposition 3.4. If the constant $h$ is fixed sufficiently small then for all $n$ sufficiently large and all $t>0$ with $\max _{i} t_{i}>c>0, P(C(n, \underline{t})) \leqslant$ $2 c_{13} 2^{-n d / \alpha} / n^{1 / 2}$ for $c_{13}$ as in Corollary 3.3.

Proof. We need only treat $n$ large, so we will in the course of the proof use various inequalities that, though not true in total generality, hold for all $n$ sufficiently large.

We take the finite set of integers $I=\left\{i_{0}, i_{1}, \ldots, i_{R}\right\}$ to be given by first taking $i_{0}$ to be the smallest integer such that $2^{i_{0}}>2.2^{-n} n^{2}$ and then 
for $1 \leqslant k \leqslant R, \quad i_{k}=i_{k-1}+\frac{8}{\alpha} \log (n)$ where $i_{R}$ is the largest such integer less than $2^{-n / 2}$. For $n$ large $R$ will be greater than $\alpha n / 18 \log (n)$.

We take $s_{k}$ to be $\left(n^{\frac{4}{\alpha}}-1\right) 2^{i_{k}}$ for $0 \leqslant k<R-1$ and $v_{k}=\left(c_{14} s_{k} / \log (n)\right)^{1 / \alpha}$. $c_{14}$ is a constant not depending on $n$ to be chosen later. For $n$ large we have (the fact that the choice of $c_{14}$ is still in abeyance notwithstanding) for all $0 \leqslant k<R$ that $2^{i_{k}}<s_{k}<2^{i_{k+1}}$.

We have from Proposition 3.1 that if $0 \leqslant k<R$ and $\left|\underline{X}\left(\underline{t}+2^{i_{k}}\right)\right| \leqslant v_{k}$, then

$$
\begin{aligned}
& P\left(L\left(\left[\underline{t}+2^{i_{k}}, \underline{t}+2^{i_{k}}+s_{k}\right]>c_{g}\left(s_{k}\right)^{N} /\left(v_{k}\right)^{d} \mid \mathcal{F}_{\underline{t}+2^{i_{k}}}\right)>c_{g} e^{-c_{8} s_{k} /\left(v_{k}\right)^{\alpha}}\right. \\
& =c_{g} e^{-\left(c_{8} / c_{14}\right) \log (n)} .
\end{aligned}
$$

We now fix $c_{14}$ so large that for $n$ large $c_{g} e^{-\left(c_{8} / c_{14}\right) \log (n)}>\frac{1}{n^{1 / 4}}$. With this value fixed the event $\left\{L\left(\left[\underline{t}+2^{i_{k}}, \underline{t}+2^{i_{k}}+s_{k}\right]>c_{g}\left(s_{k}\right)^{N} /\left(v_{k}\right)^{d}\right\}\right.$ can be rewritten as $\left\{L\left(\left[\underline{t}+2^{i_{k}}, \underline{t}+2^{i_{k}}+s_{k}\right]>\left(c_{g} / c_{14}^{d / \alpha}\right)\left(s_{k}\right)^{N-d / \alpha}(\log (n))^{d / \alpha}\right\}\right.$. We will now choose $h$ to be $\left(c_{g} / c_{14}^{d / \alpha}\right) / 2$ so that for $n$ large

$$
\left(c_{g} / c_{14}^{d / \alpha}\right)\left(s_{k}\right)^{N-d / \alpha}>h\left(s_{k}+2^{i_{k}}\right)^{N-d / \alpha} \quad \text { for all } 0 \leqslant k<R .
$$

To conclude the proof we note that if event $A(\underline{t}, n)$ holds, then for each $0 \leqslant k<R, \quad\left|X_{j}\left(t_{j}+2^{i_{k}}\right)-X_{j}\left(t_{j}\right)\right| \leqslant n^{2 / \alpha} 2^{k / \alpha}$ and so if $A(\underline{t}, n) \cap$ $\left\{|\underline{X}(\underline{t})| \leqslant 2^{-n / \alpha} n^{1 /(2 d)}\right\}$ holds then for all $0 \leqslant k<R$ (if $n$ is sufficiently large),

$$
\left|\underline{X}\left(\underline{t}+2^{i_{k}}\right)\right| \leqslant 2^{-n / \alpha} n^{1 /(2 d)}+N n^{2 / \alpha} 2^{k / \alpha} \leqslant v_{k} .
$$

Hence we have (with the above choice of $h$ ) that

$$
P(C(n, \underline{t})) \leqslant P\left(A(\underline{t}, n) \cap\left\{|\underline{X}(\underline{t})| \leqslant 2^{-n / \alpha} n^{1 /(2 d \alpha)}\right\}\right)+\left(1-\frac{1}{n^{1 / 4}}\right)^{R} .
$$

Given our bound for $R$, the proof is complete.

\section{HAUSDORFF MEASURE BOUNDS}

In this section, we establish Proposition 1.2. We consider for concreteness the cube $[1,2]^{N}$ but it will be clear that no loss of generality is entailed.

Consider the cube in $[1,2]^{N}, \Pi_{j=1}^{N}\left(1+\frac{i_{j}}{2^{n}}, 1+\frac{i_{j}+1}{2^{n}}\right]=H_{\underline{i}}^{n}$. Let the Lebesgue measure of the range of $\underline{X}$ on $H_{\underline{i}}^{n}$ be $R_{\underline{i}}^{n}$.

Lemma 4.1. For positive $k, P\left(R_{i}^{n} \geqslant k 2^{-d n / \alpha}\right) \leqslant C e^{-c k^{1 / N}}$ for some finite, positive $C, c$ not depending on $n, \underline{i}$. 
Proof. We consider the time components separately. Let the stopping times $\sigma_{k}^{j}$ (with respect to $\mathcal{F}_{s}^{j}=\sigma\left(\underline{X}_{j}(u): 0 \leqslant u \leqslant s\right.$ ), the natural one for the process $\left(X_{j}(s): s \geqslant 0\right)$ be defined recursively by $\sigma_{0}^{j}=1+i_{j} / 2^{n}$ and for $k \geqslant 1$,

$$
\sigma_{k}^{j}=\inf \left\{t>\sigma_{k-1}^{j}: d\left(X_{j}(t), X_{j}\left(\sigma_{k-1}^{j}\right)\right) \geqslant 2^{-n / \alpha}\right\} .
$$

Then we have by the strong Markov property and natural scaling properties, that there exists $c$ not depending on $j, n, k$ or $\underline{i}$ such that

$$
P\left[\sigma_{k}^{j}>\sigma_{k-1}^{j}+2^{-n} \mid \mathcal{F}_{\sigma_{k-1}^{j}}^{j}\right] \geqslant c
$$

$\left(\mathcal{F}_{T}^{j}\right.$ is the usual $\sigma$-field for a stopping time $T$ for the filtration $\left.\mathcal{F}_{S}^{j}\right)$ and so

$$
P\left[\sigma_{k}^{j} \leqslant 1+\frac{i_{j}+1}{2^{n}}\right] \leqslant(1-c)^{k} .
$$

Let $M_{j}^{\underline{i}}=\sup \left\{k: \sigma_{k}^{j} \leqslant 1+\frac{i_{j}+1}{2^{n}}\right\}$ then the range of $\underline{X}$ on $H_{\underline{i}}^{n}$ is contained in

$$
\bigcup_{k_{1}=0}^{M_{1}^{i}} \bigcup_{k_{2}=0}^{M_{2}^{i}} \cdots \bigcup_{k_{N}=0}^{M_{n}^{i}} A\left(k_{1}, k_{2}, \ldots, k_{N}\right)
$$

where

$A\left(k_{1}, k_{2}, \ldots, k_{N}\right)=\left\{\underline{x}_{1}+\underline{x}_{2}+, \cdots \underline{x}_{N}: \underline{x}_{j} \in B\left(\underline{X}_{j}\left(\sigma_{k_{j}}^{j}\right), 2^{-n / \alpha}\right), j=1,2, \ldots, N\right\}$, since the Lebesgue measure of $A\left(k_{1}, k_{2}, \ldots, k_{N}\right)$ is bounded by $C_{d} N^{d} 2^{-d n / \alpha}$ we easily have

$$
R_{\underline{i}}^{n} \leqslant C_{d} N^{d} 2^{-d n / \alpha}\left(M_{1}^{\underline{i}}+1\right)\left(M_{2}^{\underline{i}}+1\right) \ldots\left(M_{N}^{\underline{i}}+1\right)
$$

and so, given this bound

$$
P\left(R_{\underline{i}}^{n}>c_{d, N}\left(2^{-d n / \alpha}\right)(k+1)^{N}\right) \leqslant N(1-c)^{k}
$$

from which the result follows.

In a similar way we can show for $D_{\underline{i}}^{n}=$ diameter of range of $\underline{X}$ on $H_{\underline{i}}^{n}$.

Lemma 4.2. There exists finite, positive $C, c$ so that for all $n, \underline{i}$,

$$
P\left(R_{\underline{i}}^{n} \geqslant k 2^{-n d / \alpha}, D_{\underline{i}}^{n} \geqslant v 2^{-n / \alpha}\right) \leqslant C e^{-c k^{1 / N}} \frac{1}{v^{\alpha}} .
$$


Proof of Proposition 1.2. We must show that there is an "economical" covering of the 0 level set on $[1,2]^{N}$.

Recall the cube $H_{i}^{n}$ is $\left\{t: t_{j} \in\left[1+\frac{i_{j}}{2^{n}}, 1+\frac{i_{j}+1}{2^{n}}\right)\right\}$. We consider various cases :

(i) $\left\{\underline{X}(\underline{t}): \underline{t} \in H_{i}^{n}\right\} \cap\{\underline{0}\}=\emptyset$ (in this case the cube $H_{i}^{n}$ does not intersect the level set);

(ii) $\{0\} \subset\left\{\underline{X}(\underline{t}): \underline{t} \in H_{\underline{i}}^{n}\right\}, R_{\underline{i}}^{n} \geqslant n^{2} 2^{-d n / \alpha}$;

(iii) $\underline{0} \in\left\{\underline{X}(\underline{t}): \underline{t} \in H_{\underline{i}}^{n}\right\}, R_{\underline{i}}^{n} \leqslant n^{2} 2^{-d n / \alpha} ; D_{\underline{i}}^{n} \geqslant n^{\frac{1}{2 d}} 2^{-n / \alpha}$;

(iv) $\underline{0} \in\left\{\underline{X}(\underline{t}): \underline{t} \in \bar{H}_{\underline{i}}^{n}\right\}, R_{i}^{n} \leqslant n^{2} 2^{-n / \alpha}, D_{\underline{i}}^{n}<n^{\frac{1}{2 d}} 2^{-n / \alpha}$ but there does not exist $n^{2} 2^{-n} \leqslant s \leqslant 2^{-n / 2}$ so that

$$
L\left(\Pi_{j=1}^{N}\left[1+\frac{i_{j}}{2^{n \alpha}}, 1+\frac{i_{j}}{2^{n \alpha}}+s\right]\right) \leqslant \frac{h}{2} s^{N-d / \alpha}\left(\log \log \left(\frac{1}{s}\right)\right)^{d / \alpha}
$$

where $h$ is small as in Proposition 3.4,

(v) all other cases.

Then we have by Lemma 4.1 that the probability that for cube $H_{i}^{n}$ (ii) occurs is bounded by

$$
\begin{aligned}
& \sum_{k=0}^{\infty} P\left(\underline{X}(\underline{t})=\underline{0} \text { for some } \underline{t} \in H_{\underline{i}}^{n} \mid R_{\underline{i}}^{n} \in\left[2^{k} n^{2} 2^{-d n / \alpha}, 2^{k+1} n^{2} 2^{-d n / \alpha}\right)\right. \\
& \times P\left(R_{i}^{n} \in\left[2^{k} n^{2} 2^{-d n / \alpha}, 2^{k+1} n^{2} 2^{-d n / \alpha}\right)\right) \\
& \leqslant \sum_{k=0}^{\infty} P\left(R_{\underline{i}}^{n} \geqslant 2^{k} n^{2} 2^{-d n / \alpha}\right) P\left(\underline{X}(t)=\underline{0} \text { for } \underline{t} \in H_{\underline{i}}^{n} \mid R_{\underline{i}}^{n} \in\left[2^{k} n^{2} 2^{-d n / \alpha},\right.\right. \\
& \\
& \left.\left.2^{k+1} n^{2} 2^{-d n / \alpha}\right)\right) \\
& \leqslant \sum_{k=0}^{\infty} C e^{-c 2^{k / N} n^{2 / N}} 2^{k+1} n^{2} 2^{-d n / \alpha} \\
& \leqslant C e^{-c n^{2 / N}} n^{2} 2^{-d n / \alpha} \leqslant C e^{-c n^{2 / N}} 2^{-n d / \alpha} \text { for } c, C \text { not depending on } n, \underline{i} .
\end{aligned}
$$

Here the inequality $P\left(\underline{X}(\underline{t})=\underline{0}\right.$ for some $\underline{t} \in H_{\underline{i}}^{n} \mid R_{\underline{i}}^{n} \in\left[2^{k} n^{2} 2^{-d n / \alpha}\right.$, $\left.2^{k+1} n^{2} 2^{-d n / \alpha}\right) \leqslant C 2^{k} n^{2} 2^{-d n / \alpha}$ follows from the fact that the random variable $R_{i}^{n}$ is independant of $\underline{X}\left(1+\frac{i}{2^{n}}\right)$ which has density bounded by a constant. 
For case (iii) we have, similarly, by Lemma 4.2 that the probability (uniformly on $H_{\underline{i}}^{n}$ ) that (iii) holds is bounded by

$$
\begin{aligned}
\sum_{k=0}^{2 \log _{2}(n)}( & P\left(\underline{X}(\underline{t})=\underline{0} \text { for some } \underline{t} \in H_{\underline{i}}^{n} \mid R_{\underline{i}}^{n} \in\left[\left(2^{k}-1\right) 2^{-d n / \alpha}, 2^{k+1} 2^{-d n / \alpha}\right),\right. \\
\left.D_{\underline{i}}^{n}>2^{-n / \alpha} n^{1 / 2 d}\right) & \\
\times & \left.P\left(R_{\underline{i}}^{n} \in\left[\left(2^{k}-1\right) 2^{-d n / \alpha}, 2^{k+1} 2^{-d n / \alpha}\right), D_{\underline{i}}^{n}>2^{-n / \alpha} n^{1 / 2 d}\right)\right) \\
\leqslant & \sum_{k=0}^{2 \log _{2}(n)} C^{\prime} 2^{k+1} 2^{-d n / \alpha} e^{-c 2^{k / N} n^{2 / N}} / n^{\alpha / 2 d},
\end{aligned}
$$

which is less than $C 2^{-n d / \alpha} \frac{1}{n^{\alpha / 2 d}}$ for some $C$ not depending on $n$ or $\underline{i}$.

We have by Proposition 3.4, that the probability that $H_{\underline{i}}^{n}$ belongs to case (iv) is bounded by $C 2^{-n d / \alpha} / n^{1 / 2}$.

We now define the cubes $V_{\underline{i}}^{n}$ by

$=\emptyset$ if (i) holds

$=H_{i}^{n}$ if (ii), (iii) or (iv) hold. This collection of $\underline{i}$ will be denoted $B^{n}$, $=\left[1+\frac{i_{1}}{2^{n}}, 1+\frac{i_{1}}{2^{n}}+s\right] \times\left[1+\frac{i_{2}}{2^{n}}, 1+\frac{i_{2}}{2^{n}}+s\right] \times \cdots\left[1+\frac{i_{N}}{2^{n}}, 1+\frac{i_{N}}{2^{n}}+s\right]$ otherwise, where $s$ is the smallest element of $\left[n^{2} 2^{-n}, 2^{-n / 2}\right]$ so that

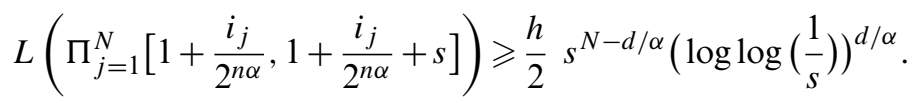

Let $J^{n}$ be the (random) collection of $\underline{i}$ so that for cube $H_{i}^{n}$ condition $(v)$ holds. For $i \in J^{n}$, the cube $E_{i}^{n}$ will be the cube with the same centre as $V_{i}^{n}$ but with a side length five times as large. Then by the usual Vitelli covering argument, we have that there exists $I^{n} \subset J^{n}$ so that

(i) for distinct $i, j$ in $I^{n}, V_{\underline{i}}^{n} \cap V_{j}^{n}=\emptyset$;

(ii) $\cup_{i \in J^{n}} V_{\underline{i}}^{n} \subset \cup_{i \in I^{n}} E_{\underline{i}}^{n}$.

Now the 0 level set in $[1,2]^{N}$ is contained in

$$
\cup_{i \in B^{n}} V_{\underline{i}}^{n} \cup \cup_{i \in I^{n}} E_{\underline{i}}^{n} .
$$

For a constant $K$ not depending on $n$ we have

$$
E\left[\sum_{i \in B^{n}} \phi\left(\left|D_{\underline{i}}^{n}\right|\right)\right] \leqslant K 2^{N n} \frac{2^{-n d / \alpha}}{n^{1 / 2} \wedge n^{\alpha / 2 d}} 2^{-n(N-d / \alpha)} \log (n) \rightarrow 0
$$


as $n \rightarrow \infty$. On the other hand we have that for any strictly positive $\epsilon$,

$$
\begin{aligned}
& \sum_{i \in I^{n}} \phi\left(\left|E_{\underline{i}}^{n}\right|\right) \leqslant 5^{N-d / \alpha} \sum_{i \in I^{n}} \phi\left(\left|V_{\underline{i}}^{n}\right|\right) \\
& \leqslant \frac{2.5^{N-d / \alpha}}{h} \sum_{i \in I^{n}} L\left(V_{\underline{i}}^{n}\right) \leqslant \frac{2.5^{N-d / \alpha}}{h} L\left([1-\epsilon, 2+\epsilon]^{N}\right)
\end{aligned}
$$

(Where the second inequality is derived from the disjointness of the $V_{i}^{n}$.) By continuity of the local time on intervals this latter term, for $\epsilon$ sufficiently small will be less than or equal to $\frac{2.5^{N-d / \alpha}}{h} L\left([1-\epsilon, 2+\epsilon]^{N}\right)$ if the local time is strictly positive and less than any desired psoitive constant otherwise. Thus, by Fatou's Lemma, Proposition 1.2 holds.

\subsection{Extensions}

If stable processes which are not necessarily strict are considered, then things become a little more complicated. The arguments of Ref. 12 will still go through if drift is added to the processes. However for our arguments to essentially still go through, we would have to consider $\alpha \geqslant 1$. In $\alpha=1$, there is still an appropiate scaling relation. In dealing with $\alpha>1$ we have that in arriving at Proposition 2.1 one cannot use scaling as before and in fact there is not a set $A$ so that to achieve a truncated process $Y^{v}$ (.) of mean zero one "unsuppresses" the jumps in $v A$ but in fact as the spatial scale $v$ tends to zero the perturbation required to deal with the extra drift term has an effect tending to zero. The calculations proceed after this much as in the strict stability case. If, however, the parameter $\alpha$ is less than one then one can directly employ the arguments of Section 2 to arrive at a process $X^{v}$ (.) of zero mean: the drift term locally dominates and it is not possible simply to alter the jump rates for jumps of order $v$ to create a process of zero mean. In this case, we not only cannot show results analogous to Theorem 1.1, we suspect that they may no longer pertain: if the (uni-dimensional time)process has a strong tendency to a given direction then the creation of a large local time will be a more complex event than simply independent recurrent behaviour for the uni-dimensional processes.

\section{ACKNOWLEDGMENTS}

The research of this author is partially supported by the NSF Grant DMS. The author wishes to thank Davar Khoshnevisan for advice, rewarding discussions and suggestions. 


\section{REFERENCES}

1. Ethier, S. N., and Kurtz, T. G. (1986). Markov Processes: Characterization and Convergence, Wiley, New York.

2. Falconer, K. J. (1985), The Geometry of Fractal Sets, Cambridge University Press, Cambridge.

3. Geman, D., and Horowitz, J. (1980). Occupation densities. Ann. Probab. 8, 1-67.

4. Khoshenevisan, D., Xiao, Y., and Zhong, Y. (2003). Local times of additive Lévy processes, I: Regularity. Stochastic Process. Appl. 104, 193-216.

5. Khoshenevisan, D., and Xiao, Y. (2002). Local times of additive Lévy processes, I: Regularity. Ann. Probab. 30(1), 62-100.

6. Liggett, T. M. (1985). Interacting Particle Systems, Springer, New York; Wiley, New York.

7. Mountford. T., and Nualart, E. (2004). Level sets for additive Brownian motion. Electron. J. Probab. 1, 138-163.

8. Mueller, C., and Tribe, R. (2002). Hitting properties of a random string. Electron. J. Probab. 7, 1-29.

9. Orey, S., and Pruitt, W. E. (1973). Sample functions of the $N$-parameter Wiener process. Ann. Probab. 1, 138-163.

10. Rogers, C. A. (1998). Hausdorff Measures, Cambridge University Press, Cambridge.

11. Samorodnitsky, G., and Taqqu, M. (1994). Stable Non-Gaussian Random Processes, Chapman and Hall, London.

12. Xiao, Y. (1997). Hölder conditions for the local times and the Hausdorff measure of the level sets of Gaussian random fields. Probab. Theorey Relat Fields 109, 129-157. 